\title{
PERIOSTIN - A FORWARD STEP IN PERIODONTAL BIOMARKERS - A NARRATIVE REVIEW
}

\section{Dr. R. Reshmaa, Dr. Sabitha Sudarsan, Dr. U. Arunmozhi, Dr. R. Kadhiresan, Dr. R. A. Jenifer Cynthia}

Department of Periodontology and Oral Implantology, Sri Venkateswara Dental College and Hospital, Thalambur, Chennai, India

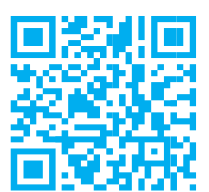

DOI: $10.37841 /$ jidam_2021_V8_I3_04

Address for Correspondence

Dr. R. Reshmaa,

Post Graduate Student, Department of Periodontology and Oral Implantology

Sri Venkateswara Dental College and Hospital Off OMR road, Near Navalur, Thalambur, Chennai-603103

E-mail: reshmaa.rajendran10696@gmail.com

Received: 12.06.2021

Accepted: 26.08.2021
First Published: 02.09.2021

Published: 27.09.2021

\section{ABSTRACT}

Various biomarkers of periodontal disease are involved in either disease pathogenesis or tissue damage. The main aim of biomarkers research in Periodontology is to provide a significant impact on clinical diagnosis as well as treatment outcomes. Periostin, a microcellular protein is one of the novel biomarkers which is expressed mostly in collagen rich tissues. Its role in systemic health and disease has been studied for many years. Periostin plays a major role in collagen development, cell migration, wound healing and repair and also regulates periodontal pathogenesis. This review focuses on the different aspects of periostin in periodontal health and disease.

Keywords: Periostin, Biomarker, Periodontitis, Periodontal regeneration 


\section{Reshmaa et al: Periostin - A Forward Step In Periodontal Biomarkers - A Narrative Review}

\section{INTRODUCTION:}

Extracellular matrix (ECM) is an extensive highly dynamic structural and molecular network. The connective tissue is composed of fibroblast and extracellular matrix which contains three major components: proteoglycans, glycosaminoglycan, and glycoproteins. ${ }^{1}$ Components of extracellular matrix (ECM) and cell adhesion receptors interact with each other and forms a complex network in all tissues. These Cell surface receptors transfers signals into cells from ECM, which aids in regulating diverse cellular functions, such as survival, growth, proliferation, migration, differentiation, wound repair and some major role in maintaining cells homeostasis. ${ }^{2}$

ECM also contains "Matricellular proteins" which are a group of proteins contributing indirectly to the formation of structural elements. They also serve to catalyze cell to cell, cell to matrix interactions and cell functions. These Matricellular proteins has a distinct characteristic to bind to many cell surface receptors, growth factors, cytokines, proteases and components of the ECM. ${ }^{3}$ They are expressed during development and injury in high levels. The Matricellular proteins are; osteopontin; periostin; Secreted Protein Rich Acidic and Rich in Cysteine SPARC family members; tenascin-C; thrombospondins1 and 2; WISP1, 2, and 3; Nov; galectin 1, 2, 3, 4, 8, and 9; Cyr61; and bone sialoprotein.

One such essential matricellular protein is Periostin (POSTN) found in $1993 .{ }^{4}$ Periostin helps not only in bone formation and regulation but also is essential for connective tissue integrity by helping in cell activation and cell binding in both health and disease. In systemic health, Periostin aids in the formation of endogenous cardiomyocytes, tendon cells. It also has a negative role in bronchial asthma where increased periostin levels cause airway obstruction by fibrosis. It also has a major function in tumor genesis by promoting cell proliferation and adhesion. Present in adipose tissues, periostin helps in the repair of subcutaneous fat in both obesity and diabetes patients. Interestingly, there are studies showing that Periostin has a major role in periodontal tissue formation (Figure 1) and regeneration. ${ }^{5}$ The aim of this review article is to provide an extensive assessment of the implications of Periostin in periodontal tissue biology and its potential use as a biomarker and also in periodontal tissue regeneration.

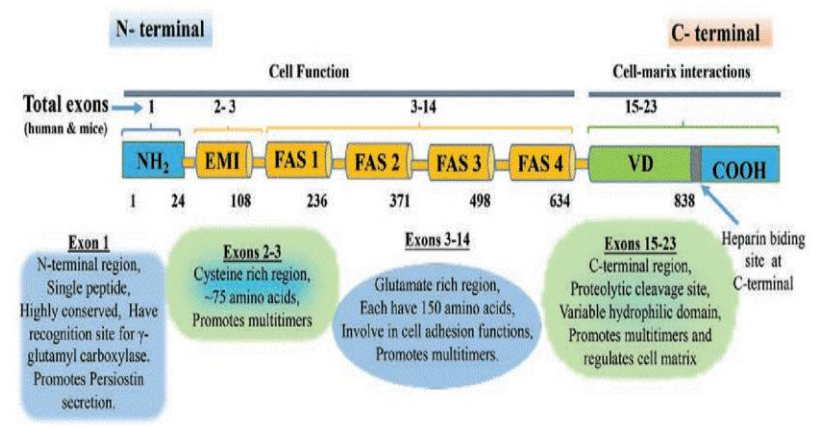

Fig 1: Functions of Periostin

\section{STRUCTURE OF PERIOSTIN:}

Periostin was originally named as Osteoblast Specific Factor 2 (OSF-2) and was later recoined as Periostin due to its localisation in periosteum and Periodontal ligament (PDL). It was first identified using subtractive hybridization techniques on the mouse osteoblastic cell line MC3T3-E. It is a $90 \mathrm{kDa}$ glutamate-containing secreted matricellular protein. Periostin is most commonly expressed in collagen-rich fibrous connective tissues which are subjected to constant mechanical strains. It is generally present in periosteum, PDLs, tendons, heart valves, and skin. ${ }^{6}$ Periostin may be involved in tissue remodeling by promotion of adhesion, cellular differentiation, cell survival, and fibrogenesis. Secreted by fibroblasts, Periostin is found to be present in serum, saliva and also GCF.

Periostin is a member of Fasciclin-I family and the family of fasciclin genes consists of four highly allied members $^{7}$ : Periostin, bIG-H3, Stabilin-1 and stabilin-2. Periostin constitutes of an amino-terminal in the EMILIN family (EMI domain), a tandem repeat of four Fas I domains (RD 1-4), a carboxyl-terminal region (CTR), and a heparin binding site at its $\mathrm{C}$-terminal end. (Figure 2)

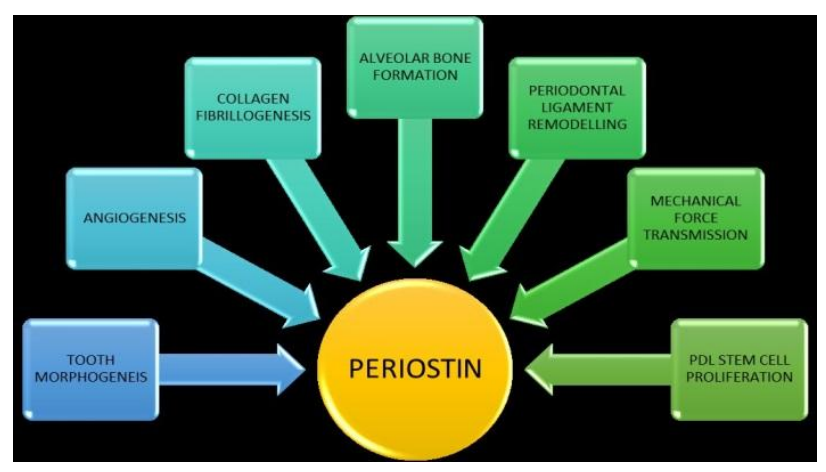

Fig 2: Structure of Periostin

1) Fas-1 domain - Helps in cell adhesion by interacting with Tenascin- $\mathrm{C}$ and Bone morphogenetic protein (BMP-1). Each Fas-1 domain has glutamate residues and an $\mathrm{N}$-terminal recognization site for vitamin $\mathrm{K}$ - 


\section{Reshmaa et al: Periostin - A Forward Step In Periodontal Biomarkers - A Narrative Review}

dependent $\gamma$-glutamyl carboxylase which is modified into $\gamma$-carboxyglutamate post-translationally.

2) EMI domain - Helps in protein to protein interaction by directly interacting with type I collagen and Fibronectin

3) C-terminal domain - Helps in producing human isoforms of Periostin by splitting alternatively. ${ }^{8}$

\section{ROLE OF PERIOSTIN IN DENTAL TISSUES:}

\section{Periostin in tooth development:}

In tooth germ, Periostin is recognizable at the interface between inner enamel epithelium and preodontoblasts and in the mesenchymal tissues around the cervical loop at the cap stage. During bell stage, Periostin is expressed in dental follicles and surface of the alveolar bone. After birth, Periostin is intensely seen in the Periodontal ligament. ${ }^{9}$ Studies with Periostin null mice had reduced cartilaginous growth plates. Incisors had widened PDLs and abnormal alveolar bone remodeling and destruction. The structurally unstable PDL has lost its mechanical properties leading to rapidly progressive periodontitis like disorder. Defects in ameloblast morphology and function resulted in secretion of inappropriate amorphous matrix and abnormal enamel and dentin structure, ultimately resulting in enhanced tooth wear due to enamel and dentin matrix defects. Thus, it was shown that periostin is critically important in maintaining the integrity of PDLs and is essential for postnatal development as it controls the morphogenesis of the tooth development. ${ }^{10}$

\section{Periostin in Angiogenesis:}

Periostin facilitates angiogenesis by its Fas1-2 domain which helps in cell adhesion. It promotes capillary formation in a concentration dependent manner by significantly enhancing vasculo endothelial growth factor receptor -2. Periostin regulates angiogenesis by upregulation of Matrix metalloproteinase's MMP-2, which also stimulates VEGF receptor -2 . Thereby periostin aids in wound healing and repair during periodontal remodelling, post periodontal surgery and regenerative procedures. ${ }^{11}$

\section{ROLE OF PERIOSTIN IN PERIODONTAL}

\section{HEALTH:}

\section{Periostin in collagen formation:}

Collagen fibrillogenesis is a complex multi-step process and matricellular proteins play a major role in collagen assembly. Many studies confirm the colocalisation and molecular interaction between periostin and Type I collagen. Periostin interacts with BMP-1 and aids in the formation of high stiffness collagen by enhancing collagen cross-linking by proteolytic activation of lysosyl oxidase (LOX). It also helps in maintaining collagen fibrils diameter, collagen maturation and assembly and preserves the biomechanical properties of connective tissue. ${ }^{12}$

\section{Periostin in gingiva:}

Immunohistochemical studies show that Periostin is present in relatively higher levels around the gingival collagen fibres of the non-bone attached portion of the periodontal ligament. It is also present just beneath the epithelium as it is associated with some basement membrane components and it is co-localised with type I collagen. It was not found within the cytoplasm of basal epithelial cells. Periostin plays a major role in the interactions of the connective tissues with the epithelial cells. $^{13}$

\section{Periostin in bone formation:}

Periostin is known to play a vital role in osteoblast adhesion, differentiation, and survival, recruitment and attachment. ${ }^{14}$ Periostin promotes the expression of type I collagen, osteocalcin, osteopontin and alkaline phosphatase by osteoblast. Thus, Periostin has a role in maintaining bone microarchitecture and bone strength. There is currently no evidence that Periostin is expressed in osteoclasts of alveolar bone. In osteocyte it is an immature osteocyte marker. In periostin null mice, there is an increased secretion of sclerostin which causes bone loss and increased apoptosis of osteoblast and osteocyte. Thereby, Periostin acts as an anti-apoptotic factor. ${ }^{15}$

\section{Periostin in Mechanical loading and force}

\section{transmission:}

Periodontal ligament transmits force resulting from mastication. This mechanical strain activates transforming growth factor beta which in turn increases periostin mRNA levels and activates periostin which helps in maintaining the integrity of PDL fibre in response to mechanical stress. ${ }^{16}$ Periostin is involved in periodontal remodelling in response to mechanical stress by orthodontic tooth movement. Divergent expression of Periostin mRNA is seen strongly in the pressure sites than in tension sites after 3 to $96 \mathrm{hrs}$ of tooth movement. Sclerostin levels also increases after tooth movement in the pressure sites. This suggests that there is an interaction between Periostin and Sclerostin during tooth movement which aids in bone remodelling. ${ }^{17}$

\section{ASSESSMENT OF PERIOSTIN LEVELS:}

Periostin is expressed in serum, saliva and GCF which can be used to determine disease and health. It can be quantitatively and qualitatively measured by various diagnostic methods such as ELISA, quantitative real time PCR, mRNA analysis. ${ }^{14}$ 


\section{Reshmaa et al: Periostin - A Forward Step In Periodontal Biomarkers - A Narrative Review}

\section{PERIOSTIN IN PERIODONTAL DISEASE:}

Periostin in gingivitis:

Studies shows that the total amount and concentration of periostin decreased in gingival crevicular fluid with the progression and severity of the disease. Periostin levels declined from healthy controls to gingivitis and to chronic periodontitis groups. However, there was no significant difference in serum periostin concentration within all groups. ${ }^{18}$

\section{Periostin in gingival enlargement:}

Studies show that periostin up-regulation is associated with gingival enlargement in patients on systemic administration of nifedipine. ${ }^{19}$ Similarly, elevated levels of periostin were seen in Phenytoin induced gingival enlargement ${ }^{20}$ where it enhances the accumulation of ECM rather than fibrosis. In Drug induced enlargement, there is an up-regulation of TGF- $\beta$ which in turn increases periostin levels despite the inflammation.

\section{Periostin in Chronic Periodontitis:}

Periodontitis is caused by bacteria acting in a preferentially susceptible host. Inflammatory mediators and cytokines released causes attachement loss and bone loss by collagen destruction. This affects the matricellular proteins and the PDL integrity. Tumour Necrosis Factor $\alpha$ (TNF- $\alpha)$ and LPS initially might increase the periostin levels in fibroblasts, but chronic exposure leads to decreased levels of periostin. ${ }^{21}$ Periostin levels negatively correlated with all the site-specific clinical paramters whereas TNF- $\alpha$ positively correlated in patients with both chronic periodontitis and diabetes. ${ }^{22}$ Similarly, periostin levels in saliva was evaluated and the results showed that the level of periostin in saliva in patients with chronic periodontitis was significantly lower than healthy controls and Periostin was detectable in all samples. They concluded that there is a significant relationship between the level of periostin in saliva and chronic periodontitis. ${ }^{23}$

\section{Periostin in Aggressive Periodontitis:}

Aral et al. ${ }^{24}$ evaluated the levels of POSTN in Chronic Periodontitis (CP) and Aggressive Periodontitis $(\mathrm{AgP})$ compared to non-periodontitis. Individuals were submitted to GCF and saliva sampling. The mean GCF level of POSTN was lowest in the $\mathrm{AgP}$ group as compared to the other groups and was lower in the $\mathrm{CP}$ group as compared to the healthy group. Periostin levels were increased in the saliva of patients with $\mathrm{AgP}$ as compared to the $\mathrm{CP}$ and healthy groups. Study by Jamesha et $\mathrm{al}^{25}$ also showed similar results where GCF Periostin levels decreased with increased severity of the disease. Subjects with Aggressive periodontitis had the lowest level of periostin in GCF followed by Chronic periodontitis subjects and healthy controls.

\section{Periostin in Peri-implant sulcular fluid:}

Analysis of periostin levels in Peri-implant sucular fluid and gingival crevicular fluid showed that there was no significant difference between implant and natural teeth groups respectively. ${ }^{26}$

\section{PERIOSTIN IN PERIODONTAL}

\section{REGENERATION:}

Bacterial products and inflammatory cytokines may reduce periostin levels, which in turn compromise the structure and function of the periodontium by reducing its structural and biomechanical properties. Periostin increases cell migration, recruitment, and attachment into the healing areas of different tissues.

High expression of Periostin in GCF samples of chronic periodontitis patients after 2 weeks of scaling and root planning was found. ${ }^{27}$ Furthermore, Periostin levels were used to assess the outcome of Non-surgical periodontal therapy. Studies shows that periostin levels increased after Low level laser therapy (LLLT). ${ }^{28}$ Also, Periostin levels increased after periodontal surgery in GCF of periodontitis patient. This is due to the decrease in the inflammatory stimuli and bacterial challenge which enhances periostin production thereby promoting periodontal healing and regeneration. Bone formation during mechanical loading is added by periostin and has direct implication on the immediate functional loading of dental implants. ${ }^{29}$

Periostin may function as an intermediate regulator of mechanotransduction that controls stem cell fate by upregulating stem cell markers like NANOG and OCT4 in response to force. It is also proposed that light optimal compressive force plays a role in maintaining homeostasis in periodontal tissue. These findings support the orthodontic tooth movement theory, which suggest that an optimal force can induce maximum tooth movement by promoting healing without damaging periodontium. ${ }^{30}$ Periostin acts a critical modulator of cell proliferation and remodeling during would healing in Periodontal ligament by inducing the proliferation of human Periodontal ligament stem cells (hPDSC). It shows that sufficient concentration of POSTN may effectively promote would healing in both periodontal tissues and around dental implants. ${ }^{31}$

\section{FUTURE DIRECTIONS:}

Since periostin is essential for cardiac repairing after an acute myocardial infarction, a trial of treatment with recombinant periostin in a preclinical infarct model was 


\section{Reshmaa et al: Periostin - A Forward Step In Periodontal Biomarkers - A Narrative Review}

initated. Transfer of periostin-overexpressing mesenchymal cells, resulted in the improved cardiac function. ${ }^{32}$ Recently, biomimetic scaffolds loaded with recombinant Periostin has also been used in the treatment of tendonitis where periostin helped in tissue repair and regeneration. ${ }^{33}$ Thus, diagnostic or clinical applications of anti-periostin antibody or recombinant periostin may promote further development of periostin for these applications in the dental field. Particularly in Periodontitis, application of recombinant periostin might serve as a healing factor or promoter.

\section{CONCLUSION:}

Periostin plays a fundamental role in postnatal development and repair of bone and tooth-related structures. Periostin may be involved in healing after post-periodontal surgery, regenerative procedures, or even after placement of dental implants. By promoting the migration of fibroblasts and osteoblasts, periostin may play a key role in remodeling of the PDL and surrounding bone after periodontal surgery with (or without) various biomaterials used for regeneration. Expression of protein levels of periostin may help in identifying various pathways in healing and/or disease pathogenesis in the periodontium. Periostin can act as a novel biomarker in the near future with respect to the pathogenesis of periodontitis. Usage of an anti-periostin antibody or mainly recombinant periostin may be considered for therapeutic applications in dentistry and for periodontal regeneration. More studies are needed to confirm this hypothesis and develop treatment protocols based on these scientific evidences.

\section{FINANCIAL SUPPORT AND SPONSORSHIP}

Nil

\section{CONFLICTS OF INTEREST}

There are no conflicts of interest.

\section{REFERENCES:}

1. Kusindarta, D. L., \& Wihadmadyatami, H. The Role of Extracellular Matrix in Tissue Regeneration. Tissue doi:10.5772/intechopen.75728

2. Mariotti A. The extracellular matrix of the periodontium: Dynamic and interactive tissues. Periodontology 2000 1993; 3:39-63.

3. Bornstein P, Sage H. Matricellular proteins: Extracellular modulators of cell function. Curr Opin Cell Biol 2002;14:608-616.

4. Takeshita S, Kikuno R, Tezuka K, Amann E. Osteoblast-specific factor 2: Cloning of a putative bone adhesion protein with homology with the insect protein fasciclin I. Biochem J 1993;294:271-278.
5. Hamilton DW. Functional role of periostin in development and wound repair: Implications for connective tissue disease. J Cell Commun Signal 2008;2:9-17.

6. Horiuchi K, Amizuka N, Takeshita S, Takamatsu H, Katsuura M, Ozawa H, Toyama Y, Bonewald LF, Kudo A Identification and characterization of a novel protein, Periostin, with restricted expression to periosteum and periodontal ligament and increased expression by transforming growth factor beta. J Bone Miner Res 1999;14(7):1239-1249. doi:10.1359/jbmr.1999.14.7.1239

7. Kii I, Nishiyama T, Li M, Matsumoto K, Saito M, Amizuka N, Kudo A. Incorporation of tenascin-C into the extracellular matrix by Periostin underlies an extracellular meshwork architecture. J Biol Chem 2010;285(3):2028-2039. doi:10.1074/jbc.M109.051961

8. Hoersch S, Andrade-Navarro MA. Periostin shows increased evolutionary plasticity in its alternatively spliced region. BMC Evol Biol 2010;10:30. doi:10.1186/1471-2148-10-30

9. Suzuki H, Amizuka N, Kii I, Kawano Y, NozawaInoue K, Suzuki A, Yoshie H, Kudo A, Maeda T. Immunohistochemical localization of Periostin in tooth and its surrounding tissues in mouse mandibles during development. Anat Rec A Discov Mol Cell Evol Biol 2004;281(2):1264-1275. doi:10.1002/ar.a.20080

10. Rios H, Koushik SV, Wang H, Wang J, Zhou HM, Lindsley A, Rogers R, Chen Z, Maeda M, Kruzynska-Frejtag A, Feng JQ, Conway SJ. Periostin null mice exhibit dwarfism, incisor enamel defects, and an early-onset periodontal disease-like phenotype. Mol Cell Biol 2005;25(24):11131-11144. doi:10.1128/ MCB.25.24.11131-11144.2005

11. Siriwardena BS, Kudo Y, Ogawa I, Kitagawa M, Kitajima S, Hatano H, Tilakaratne WM, Miyauchi M, Takata T. Periostin is frequently overexpressed and enhances invasion and angiogenesis in oral cancer. Br J Cancer 2006;95:1396-1403

12. Kudo A. Periostin in fibrillogenesis for tissue regeneration: Periostin actions inside and outside the cell. Cell Mol Life Sci 2011;68(19):3201-3207. doi:10.1007/s00018-011-0784-5

13. Cobo T, Obaya A, Cal S, Solares L, Cabo R, Vega JA, Cobo J. Immunohistochemical localization of periostin in human gingiva. Eur $\mathbf{J}$ Histochem 2015;59:2548

14. Romanos GE, Asnani KP, Hingorani D, Deshmukh VL. PERIOSTIN: role in formation and maintenance of dental tissues. Journal of Cellular Physiology. 2014; Jan;229(1):1-5. 


\section{Reshmaa et al: Periostin - A Forward Step In Periodontal Biomarkers - A Narrative Review}

15. Litvin J, Selim AH, Montgomery MO, Lehmann K, Rico MC, Devlin H, Bednarik DP, Safadi FF. Expression and function of Periostin-isoforms in bone. J Cell Biochem 2014;92(5):1044-1061. doi:10.1002/jcb.20115

16. Choi JW, Arai C, Ishikawa M, Shimoda S, Nakamura Y. Fiber system degradation, and Periostin and connective tissue growth factor level reduction, in the periodontal ligament of teeth in the absence of masticatory load. J Periodontal Res 2011;46(5):513521. doi:10.1111/j.1600-0765.2011.01351.x

17. Rangiani A, Jing Y, Ren Y, Yadav S, Taylor R, Feng JQ. Critical roles of Periostin in the process of orthodontic tooth movement. Eur $\mathbf{J}$ Orthod 2016;38(4):373-378. doi:10.1093/ejo/ cjv071

18. Balli U, Keles ZP, Avci B, Guler S, Cetinkaya BO, Keles GC. Assessment of periostin levels in serum and gingival crevicular fluid of patients with periodontal disease. J Periodont Res 2015; 50: 707713.

19. Kim SS, Jackson-Boeters L, Darling MR, Rieder MJ, Hamilton DW. Nifedipine induces periostin expression in gingival fibroblasts through TGF-beta. J Dent Res. 2013 Nov;92(11):1022-8. doi: 10.1177/0022034513503659. Epub 2013 Sep 4. PMID: 24004653.

20. Kim SS, Nikoloudaki G, Darling M, Rieder MJ, Hamilton DW. Phenytoin activates Smad3 phosphorylation and periostin expression in druginduced gingival enlargement. Histology and histopathology. 2018;33(12):1287.

21. Padial-Molina M, Volk SL, Rios HF. Periostin increases migration and proliferation of human periodontal ligament fibroblasts challenged by tumor necrosis factor -alpha and Porphyromonas gingivalis lipopolysaccharides. J Periodontal Res 2014;49(3):405-414. doi:10.1111/jre. 12120

22. Radhika BN, Appukuttan DP, Prakash PS, Subramanian S, Victor DJ, Balasundaram A. Estimation of Periostin and Tumour Necrosis Factor$\alpha$ in Type II Diabetics with Chronic Periodontitis: A case-control study. J Indian Soc Periodontol 2019;23:106-12.

23. Esfahrood ZR, Vardian ST, Yadegari Z, Adhim M, Saravi NS. Periostin levels in saliva of patients with chronic periodontitis. J Indian Soc Periodontol 2018;22:25-7.

24. Aral CA, Köseoğlu S, Sağlam M, Pekbağrıyanık T, Savran L. Gingival Crevicular Fluid and Salivary Periostin Levels in Non-Smoker Subjects With
Chronic and Aggressive Periodontitis : Periostin Levels in Chronic and Aggressive Periodontitis. Inflammation. 2016;Jun;39(3):986-93. doi: 10.1007/s10753-016-0328-0. PMID: 26931107.

25. Jamesha FI, Maradi AP, Chithresan K, Janakiram S, Maddur PK, Rangaraju R. Comparison of gingival crevicular fluid periostin levels in healthy, chronic periodontitis, and aggressive periodontitis. J Indian Soc Periodontol 2018;22:480-6.

26. Akman, AC, Askin, SB, Guncu, GN, Nohutcu, RM. Evaluation of gingival crevicular fluid and peri-implant sulcus fluid levels of periostin: A preliminary report. J Periodontol. 2018; 89: 195202. https://doi.org/10.1902/jop.2017.170315

27. Karim, A.F., Soeroso, Y., Sunarto, H., \& Bachtiar, B. Periostin as a periodontal healing indicator after scaling and root planing. Journal of International Dental and Medical Research, 2019;12:1143-1148.

28. Kumaresan D, Balasundaram A, Naik VK, Appukuttan DP. Gingival crevicular fluid periostin levels in chronic periodontitis patients following nonsurgical periodontal treatment with low-level laser therapy. Eur J Dent. 2016 Oct-Dec;10(4):546550. doi: 10.4103/1305-7456.195179.

29. Du J, Li M. Functions of Periostin in dental tissues and its role in periodontal tissues' regeneration. Cellular and molecular life sciences. 2017;74(23):4279-86.

30. Panchamanon P, Pavasant P, Leethanakul C. Periostin plays role in force-induced stem cell potential by periodontal ligament stem cells. Cell Biol Int 2019;43(5):506-515. doi: 10.1002/cbin.11116. Epub 2019 Mar 12. PMID: 30761669.

31. Ziqiang $\mathrm{Wu}$, Wenyong Dai, Pei Wang, Xiaozhen Zhang, Yi Tang, Lin Liu, et al. Periostin promotes migration, proliferation, and differentiation of human periodontal ligament mesenchymal stem cells, Connective Tissue Research,

2018;59:2:108-119, DOI:

10.1080/03008207.2017.1306060

32. Cho Y-H, Cha M-J, Song B-W, Kim I-K, Song H, Chang W, et al. Enhancement of MSC adhesion and therapeutic efficiency in ischemic heart using lentivirus delivery with periostin. Biomaterials 2012;33:1376-85.

33. Wang, Y., Jin, S., Luo, D. et al. Functional regeneration and repair of tendons using biomimetic scaffolds loaded with recombinant periostin. Nat Commun 12, 1293(2021). https://doi.org/10.1038/ s41467-021-21545-1 